\title{
Problem solving in cebus and rhesus monkeys
}

\author{
R. C. ELLIOTT \\ The Institute of Psychiatry, De Crespigny Park, London SE5 8AF, U.K.
}

Four rhesus and four cebus monkeys were tested on a series of five progressively more difficult latchbox problems without specific training. There was no difference between the two species on the earlier problems, but on one of the more difficult problems, the cebus monkeys were superior. Possible explanations for this difference are discussed.

The ability of cebus and rhesus monkeys to solve a graded series of latchbox problems without specific training was investigated. Cebus monkeys were thought to be particularly suitable for a study of this kind, since they have been reported to possess a high degree of manipulative skill and a wide repertoire of movements when manipulating objects (Hill, 1960; Klüver, 1933). Formal training was not given since all animals might have solved the problems with low and indistinguishable learning scores, and the experimental design thus afforded a measure of the two species' perseverance in the absence of immediate reward.

\section{METHOD}

\section{Subjects}

Four adolescent Macaca mulatta (three male, one female), ranging in weight from $2-4 \mathrm{~kg}$, and four subadult male Cebus apella, ranging in weight from $1-2 \mathrm{~kg}$, were the subjects. All animals had been adapted to training in a WGTA, but were otherwise experimentally naive.

The five latchbox problems are shown in Figure 1. The latchbox, measuring $21 \mathrm{~cm}$ wide by $14.5 \mathrm{~cm}$ deep by $14 \mathrm{~cm}$ high, was mounted centrally on the shelf of a modified WGTA. The various latches and retainers were attached to wooden blocks on the hinged door and door jamb. The hinged door formed the front face of the box, but owing to the stiffness of the hinges the door required a downward pull as the latch was opened. Most animals thus worked bimanually, although certain animals achieved a correct solution on Problems 2 and 3 merely by pulling down hard on the latch with one hand (preliminary experiments with two animals indicated that if the door was mounted on top of the box as in Hayes and Nissen, 1971, they failed to learn). The latches were graded in order of difficulty by two independent observers, and the problems were presented in that order. Peanut reward was used for correct solution.
}

\section{Procedure}

All animals were first trained on a bimanual adapting task, described elsewhere (Elliott, Ettlinger, Maccabe, \& Richardson, 1975), to a criterion of 90 out of 100 correct. On Problem 1, all animals initially received five trials with the box open for

The author is grateful to the MRC who supported this work, to Dr. G. Ettlinger for advice, to Dr. R. E. Passingham for his encouragement, and to Dr. William A. Wilson, Jr., who kindly sponsored this paper and takes full editorial responsibility for its contents.

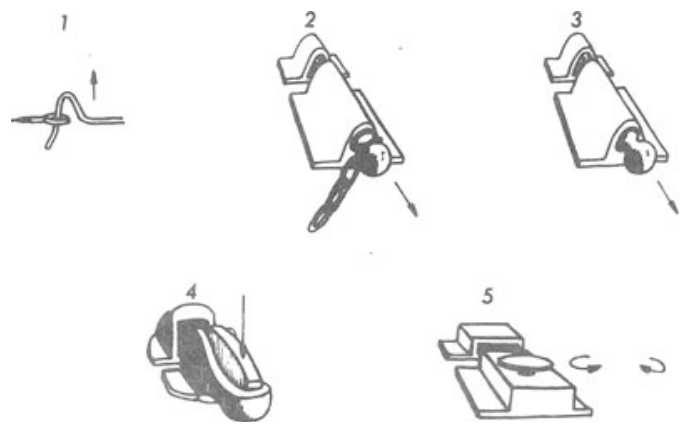

Figure 1. The five latches and retainers used in the experiment. The problems were presented to all animals in the order $1-5$, the required movement for correct solution being shown by the arrows.

adaptation. Subsequently, no further help was given. Each animal was allowed $30 \mathrm{sec}$ to solve the problem, after which the trial was terminated and the problem re-presented. After five successive failures, the lock was opened for three trials enabling the animal to obtain reward merely by pulling the door downwards. This was the only form of correction given. Training was continued on each problem to 19 out of 20 correct solutions, or for 90 trials, whichever was the sooner. Errors were recorded as: (1) incorrect manipulation of the latch, (2) failure to manipulate latch, or (3) failure to respond to apparatus at all.

\section{RESULTS}

The results are presented in Table 1 . There was no difference between the rhesus and cebus groups on the number of trials required to reach criterion on the adapting task or on Problems 1, 2, and 3 (Mann-Whitney $U$ test). Only one rhesus monkey solved Problem 4, whereas all the cebus monkeys solved this problem within 30 trials. This difference was significant on the Mann-Whitney $U$ test $(p=.01)$. Only one cebus monkey, $C_{1}$, and no rhesus monkeys solved Problem 5.

\section{DISCUSSION}

The results do not suggest that there is a substantial difference between rhesus and cebus monkeys in their ability to solve simple latchbox problems involving the cooperative use of two hands. However, the greater success of the cebus monkeys on the more difficult Problems 4 and 5 deserves attention. Observations on the 
Table 1

Number of Trials Required to Reach Criterion on the Adapting Task and Five Latchbox Problems

\begin{tabular}{lcrcccl}
\hline & Adapting & \multicolumn{5}{c}{ Problem } \\
\cline { 3 - 7 } Animals & Task & 1 & 2 & 3 & 4 & 5 \\
\hline & \multicolumn{5}{c}{ Cebus } \\
$\mathrm{C}_{1}$ & 70 & 10 & 12 & 46 & 20 & $90 \mathrm{~F}$ \\
$\mathrm{C}_{2}$ & 130 & 27 & 23 & 0 & 25 & 30 \\
$\mathrm{C}_{3}$ & 160 & 9 & 23 & 47 & 28 & $90 \mathrm{~F}$ \\
$\mathrm{C}_{4}$ & 90 & 23 & 10 & 62 & 4 & $90 \mathrm{~F}$ \\
& & & Rhesus & & & \\
$\mathrm{R}_{1}$ & 90 & 14 & 65 & 11 & $90 \mathrm{~F}$ & $90 \mathrm{~F}$ \\
$\mathrm{R}_{2}$ & 70 & 72 & 27 & 0 & $90 \mathrm{~F}$ & $90 \mathrm{~F}$ \\
$\mathrm{R}_{3}$ & 110 & 7 & 16 & $90 \mathrm{~F}$ & $90 \mathrm{~F}$ & $90 \mathrm{~F}$ \\
$\mathrm{R}_{4}$ & 170 & 50 & 0 & 40 & 59 & $90 \mathrm{~F}$ \\
\hline
\end{tabular}

Note-F indicates failed to reach criterion in the stated number of trials.

normal cage behavior of the two species indicates that whereas both possess a high degree of manipulative skill, cebus monkeys have a wider range of manipulatory movements and they will persist at some simple task (such as attempting to remove a padlock from a cage) for much longer than rhesus monkeys. Such behavior would appear to be intrinsically rewarding for them. It may be relevant that the rhesus monkeys failed to manipulate the latch at all within $30 \mathrm{sec}$ more frequently than did the cebus monkeys. Informal observations also indicated that the cebus monkeys, especially $\mathrm{C}_{2}$ and $\mathrm{C}_{4}$, spent considerably more time manipulating the latch during each trial than did the rhesus monkeys.

\section{REFERENCES}

Elliot,, R. C., Ettllinger, G., Maccabe, J. J., \& RichaRdson, N. Bi-manual motor performance in the monkey: Successive division of the forebrain and of the cerebellum. Experimental Neurology, 1976, 50, 48-59.

HAYES, K. J., \& Nissen, C. H. Higher mental functions of a homeraised chimapnzee. In A. M. Schrier \& F. Stollnitz (Eds.), Behavior of non-human primates (Vol. 4). New York: Academic Press, 1971. Pp. 60-116.

Hill, W. C. O. Primates. 4, Cebidae, Part A. Edinburgh: University Press, 1960.

KLÜVER, H. Behavior mechanisms in monkeys. Chicago: University Press, 1933.

(Received for publication December 3, 1975.) 